\title{
Uso cooperativo de Facebook en la planificación textual en la universidad
}

Cooperative use of Facebook in text planning at university

Uso cooperativo do Facebook no planejamento textual na universidade

\section{DOI: https://doi.org/10.18861/cied.2021.12.2.3040}

\section{José Miguel Cornelio Ramos}

Universidad Tecnológica del Perú

c15035@utp.edu.pe

ORCID: 0000-0002-7501-3776

Recibido: 01/12/20

Aprobado: 01/04/21

Cómo citar: Cornelio Ramos. J. M. (2021) Uso cooperativo de Facebook en la planificación textual en la universidad Cuadernos de Investigación Educativa, 12(2). https://doi.org/10.18861/cied.2021.12.2.3040

\section{Resumen}

El objetivo de esta investigación fue analizar los resultados sobre el uso cooperativo de Facebook obtenidos en una prueba de desempeño para planificar textos académicos en esquemas de redacción. La asignatura fue Nivelación de Redacción de la Universidad Tecnológica del Perú (UTP) y se trabajó con dos grupos de trabajo, cada uno constituido por 25 estuilantes instrumento de recolección de datos fue una rúbrica de tres dimensiones: contenido, estructura y formulación Los datos procesados a través delSPSS 25 (Statistical Package for Social Sciences versión 25 ) evidenciaron que en el experimento ta media obtenida en el postest alcanzó el nivel Destacado respecto al nivel Estándar del grupo de control Con ello se afrmo que el estudiantado del experimento logró utilizar ideas pertinentes. suficientes y de acuerdo con el tema elegido. De la misma manera escribió las ideas correctamente y pudo ordenarlas de forma jeŕrquica en la elaboración del esquema de redacción a través del trabajo cooperativo y autónomo aspecto que en condiciones regulares es dificil de desarrollar Por ello la investigación recomendó a dicha universidad implementar plataformas no institucionates tales como las redles sociales, amigables para el estudiantado en sus cursos de Redacción sobre todo a los que son de apertura educativa, no solo como repositorios de información y de consulta sino además como parte integral de la metodologí de la enseñanza de la escritura.

Palabras clave: escritura, redacción, tecnología de la información, medios sociales, aprendizaje en línea

\section{Abstract}

The purpose of this research was to analyze the results on the cooperative use of Facebook resulted from a performance test carried out in order to plan the academic texts on writing schemes. The subject consisted in Writing Levelling at the Technological University of Peru (UTP), where two working groups were studied: each group was made up of 25 students, The design of this research was quasi-experimental and quantitative. The sampling was dimensional rubric content structure and formulation The data processed through SPSS25 (Statistical Package for Social Sciences $v$. 25) showed that in the study for the mean obtained in the post-test an Outstanding level was reached in respect of the Standard level in the control group W/ith this it was found that students of the experiment managed to use ideas that are pertinent, sufficient and in accordance with the topic chosen In the same way, those students managed to write the ideas correctly and to order them hierarchically in the elaboration of the writing scheme, through cooperative and autonomous work -an aspect that under regular conditions is difficult to develop. For this reason, this research recommended said university to implement non-institutional platforms such as social networks -student-friendly ones- for their Writing courses, especially such social networks that are open to education, not only as repositories of information and consultation, but also as an integral part of the methodology of teaching writing

Keywords: writing, drafting, information technology, social media, online learning.

\section{Resumo}

o objetivo dessa pesquisa foi analisar os resultados sobre o uso cooperativo do Facebook obtidos mediante teste de desempenho para planejar textos acadêmicos em esquemas de escrita. A disciplina era Nivelamento em Redação da Universidade Tecnológica do

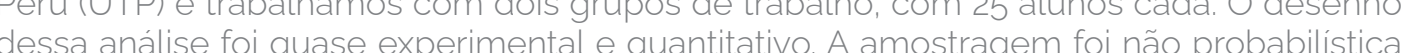
por conveniência O instrumento de coleta de dades foi uma rubrica tridimensional cujas dimensões foram conteúdo estrutura e formulação Os dados processados por meio do SPSS-25 (Statistical Package for Social Sciences versão 25) mostraram que no experimento, a média obtida no pós-teste atingiu o nivel Outstanding em relação ao nivel Padrão do grupo controle. Assim, afirmou-se que os alunos do experimento conseguiram utilizar ideias pertinentes, suficientes e de acordo com o tema escolhido. Do mesmo modo, conseguiu ordenar as ideias de maneira hierárquica e bem escrita na elaboração do esquema de redação, através do trabalho cooperativo e autônomo, aspecto que em condições normais é dificil de desenvolver. Por esse motivo, a pesquisa recomendou que a referida universidade implementasse plataformas năo instlucionais. como as edes socials, amigas do corpo discente, nos seus cursos de Redaçăo, especialmente naqueles abertos a educação, năo apenas como repositorios de informaçăo e consulta, mas tambem como parte integrante da metodologia de ensino da escrita.

Palavras-chave: escrita, redação, tecnologia da informacão, mídia social, aprendizagem online. 


\section{Introducción}

En el actual contexto de la globalización, las Tecnologías de la Información y Comunicación ( $\mathrm{TIC}$ ) son parte integral de las metodologías de la enseñanza de la escritura académica, sobre todo en ámbitos universitarios donde el aprendizaje cobra mayor autonomía y libertad. En los últimos años las redes sociales han sido vistas como recursos útiles para replantear las directrices pedagógicas tradicionales y proponer alternativas metodológicas acordes a las nuevas condiciones de socialización del siglo XXI. Por ello, Facebook ha sido la plataforma con mayores posibilidades para desarrollar conocimiento integrado sobre todo vinculado a alguna etapa del proceso de escritura (Mendoza, 2019). Esto ha sido posible no solo por su usabilidad juvenil sino además por su fácil acceso, su gratuidad y sus recursos de cooperación (Fonseca, 2015) que permiten que los usuarios puedan dar, según Garzón (2016), "rienda suelta a su imaginación y construyan conocimiento por interacción social, empleando una comunicación adaptada a la cultura de un entorno virtual, en la que prevalece lo digital con fines comunicativos, lo cual motiva a sus usuarios a expresarse libremente" (p. 141).

Pese a las virtudes de esta red social, en la enseñanza de la escritura académica se la ha excluido, ya sea por la desconfianza de muchos profesionales con respecto a las plataformas sociales digitales masivas, principalmente Facebook, ya sea por considerar a la escritura como exclusiva de claustros universitarios (Ramirez, 2013). Sea como fuere, en la Universidad Tecnológica del Perú el uso de este tipo de plataformas de acceso libre ha sido nula, y los únicos acercamientos a la apropiación de las TIC han sido mediante entornos digitales institucionales que funcionan como repositorios de contenidos, que han priorizado el trabajo individual y poco integrado al grupo. Esta condición, sumada al trabajo siempre presencial o sincrónico, al limitado tiempo para procesar información en el curso Nivelación de Redacción y, principalmente, a condiciones socioculturales del estudiantado de la universidad tales como el individualismo, ha provocado que se establecieran prácticas de rivalidad durante el aprendizaje asi como dependencia con respecto a la formación profesional, al punto de incumplir con las actividades de escritura si el docente no está a su lado (García, García y Reyes, 2014).

Frente a tal situación este estudio buscó insertar, como alternativa pedagógica, el uso de las redes sociales en todo el proceso de escritura para que el estudiantado asumiera el desarrollo de su desempeño de forma cooperativa y autónoma. Por tal razón tuvo como objetivo principal analizar los resultados del uso de Facebook obtenidos en una prueba de desempeño para planificar textos académicos en esquemas de redacción. De tal forma se buscó, en específico, identificar cómo esa plataforma afecta la calidad del contenido de la información, de la organización de las ideas y de la formulación de estas en los esquemas de redacción en el contexto del curso Nivelación de Redacción de la Universidad Tecnológica del Perú.

Para cumplir con los objetivos, la investigación en cuestión tuvo un enfoque cuantitativo y fue de diseño cuasiexperimental y explicativo. El muestreo fue no probabilístico por conveniencia, por lo que se eligió a 50 estudiantes matriculados en el periodo de estudios comprendido entre marzo y julio de 2019. Los alumnos fueron divididos en dos grupos: 25 para el controly 25 para el experimento. A estos últimos se les aplicó una prueba de escritura cuyos datos se recogieron con una rúbrica de tres niveles de diseño propio, la cual fue sometida a una validación de contenido por juicio de expertos y de constructo por análisis factorial, y se buscó su confiabilidad a través de la prueba de equivalencia mediante el grado de correlación interclase. Asimismo se realizó la prueba de normalidad con ShapiroWilk dado que fue una muestra de 25 unidades. Considerando la normalidad de los datos se realizó el contraste de hipótesis con la prueba T-Student para muestras independientes y relacionales. 


\section{Marco teórico}

Uno de los tipos de aprendizaje más efectivos para desarrollar compromiso en tareas académicas en estudiantes novatos es el cooperativo (Azorin, 2018). Este modo de comprender el aprendizaje está constituido por un agente facilitador cuya función es modelar actividades para todos los miembros del grupo designado a fin de que los integrantes puedan realizarlas bajo un objetivo común a través de interacciones que beneficiarán a todos (Johnson y Johnson, 1999). Este sistema cambia la idea de rivalidad, tan presente en entornos estudiantiles. Esto, apoyado en plataformas de la web 2.0, ofrece inagotables posibilidades para desarrollar no solo mejores actitudes para aprender sino también cualidades de solidaridad y de cooperación (Torres y Yepes, 2018). Por tal motivo puede afirmarse que los trabajos que se realizan con esta modalidad tienen un carácter social, porque para construir saberes se necesita de otros que contribuyan activamente pero bajo ciertas directrices que lo hagan coherente (Azorín, 2018).

El conocimiento significativo construido se obtiene en virtud de cinco aspectos puntuales. Según Vivero, Toala y Macias (2018), en primer lugar, la interdependencia positiva se entiende como aquella dependencia entre los integrantes de un grupo que tienen como finalidad resolver actividades bajo objetivos comunes y un sentido de hermandad. Esta forma de trabajo permite que el grupo pueda coordinar acciones en beneficio del grupo. El segundo aspecto a considerar es la interacción que se realiza a través de intercambios verbales. A través de ello el grupo se retroalimenta, intercambia ideas y propone formas diversas de resolver los problemas. El tercero hace referencia a la evaluación no solo individual sino además grupal. Si bien cada integrante del grupo es responsable por alguna actividad en específico, todo el grupo lo es también porque es su rol apoyar a quien no puede cumplir. El cuarto se refiere a las habilidades sociales utilizadas para la cooperación. Este modo involucra expresiones personales que respeten la diversidad de todo tipo. La última caracteristica del aprendizaje cooperativo es el procesamiento del grupo, lo que significa que se resalta el modo en que el grupo gestionó sus roles para cumplir con la actividad.

Según Alarcón, Sepúlveda y Madrid (2018) este tipo de aprendizaje se diferencia de otras formas educativas más frecuentes en el aula tales como el trabajo en grupo y el colaborativo. En principio, trabajar en grupo no significa hacerlo cooperativamente. La agrupación de integrantes bajo una actividad común no garantiza que se trabaje coordinadamente y se evite que un integrante pueda hacerlo individualmente. La cooperación implica una estructura pautada bajo objetivos y roles. Respecto al aprendizaje colaborativo, la diferencia es más compleja e integral. La diferencia mayor es que lo colaborativo es una filosofía personal. En cambio, lo cooperativo es una técnica de trabajo en grupo, por lo que el aprender cooperativamente es aprender colaborativamente, pero no a la inversa.

Uno de los modelos más interesantes en entornos universitarios es el Co-op co-op (técnica flexible de aprendizaje cooperativo) propuesto por Kagan (1985). Según Fernández de Haro (2013) este método tiene como objetivo que el alumnado se involucre a través de relaciones de cooperación con las actividades individuales y de grupo. Concretamente se desarrolla en tres pasos. Primero, al alumnado se le entrega el trabajo, es decir, se presenta la actividad y se definen los objetivos de grupo. Segundo, cada grupo trabaja bajo directrices autónomas pero considerando los objetivos individuales y del grupo. Tercero, todos los productos se socializan para que sean valorados.

Existen varias plataformas virtuales que permiten trabajar de esta forma. Sin embargo, las redes sociales son ideales para estos tipos de trabajo, precisamente 
en esta era de la globalización porque, en principio, son gratuitas, flexibles en su uso e interactivas (Del Barrio y Ruiz, 2014). Por esta razón Facebook, como plataforma con esas características, además de ser muy amigable a los ojos del escritor novato, es muy usada para desarrollar conocimiento cooperativo pues cuenta con excelentes recursos relacionados con el trabajo en equipo que pueden ayudar a promover participación inclusiva y creativa y de fortalecimiento de la comunicación (Simanca, Blanco y Cifuentes, 2019). Varias investigaciones sobre su aplicación en la educación lo demuestran.

Ejemplos de estos usos exitosos son las investigaciones de Gudiño, Lozano y Fernández (2014), quienes afirman que la plataforma es un instrumento mediador entre el docente y los estudiantes. La dinámica de socialización de este entorno permitió que los participantes pudieran aprender, en este caso, una segunda lengua. Asimismo la investigación de Rueda y Giraldo (2016) enfatizó en las cualidades innovadoras de Facebook pero agregó algo más: la forma de diferenciación que, basada en un nuevo sistema de comunicación, permitió que cada usuario pudiera imprimir su marca identitaria como escritor en la web a través de fotos que generaran una singularidad distinta y única. La imagen en estos entornos permite una reflexión permanente del escritor en web. Por su parte, a partir de una investigación de corte cualitativo Álvarez (2017) analizó e interpretó la relación entre el uso de esta plataforma con la modificación del lenguaje académico. Mencionó que, incluso, esta forma de escritura cuestionó el lenguaje popular, cotidiano. La consideró sumamente innovadora porque, gracias a la interactividad que promueve la plataforma se construyen nuevos modos de expresión.

También Aristizábal, Becerra y Díaz (2018) resaltaron esa misma idea al considerar que la programación cooperativa, durante cinco semanas, mejoró la ortografía, el léxico y la escritura gramatical en un grupo de estudiantes jóvenes en Colombia. Del mismo modo Núñez et al. (2020) afirmaron que esta red, a través de los comentarios en los foros, permite una libre competencia de opiniones valorativas. De esa manera, escribir en Facebook es construirse una identidad como escritor. No es un acto aislado ni individual, sino colectivo.

Por ese motivo, esta plataforma ha sido muy aceptada por investigadores universitarios, precisamente por su flexibilidad, por su capacidad para el trabajo en línea y la interacción de forma eficiente, pues, según González (2012),

resulta un espacio virtual idóneo para propiciar el aprendizaje cooperativo. Docentes y alumnos pueden encontrarse y seguir compartiendo todo lo tratado en el aula, ideas, problemas, etc. Por esta razón, no cabe duda de que Facebook puede ser una plataforma en la que los docentes propongan proyectos, trabajos en grupos, investigaciones y un sin par de actividades didácticas que han de ser realizadas con el esfuerzo y la ayuda de todos los alumnos, compartiendo conocimientos, ideas, habilidades, etc. (p. 70)

En ese sentido es un entorno en el que se puede trabajar cooperativamente, es decir, se aprende con una metodología que fomenta el trabajo compartido e interactivo, de ahi que la comunidad cientifica considere que este tipo de metodología se potencia con los grupos de Facebook (Parra, González-Sicilia y Beltrán, 2013). Un recurso ideal para trabajar en esta plataforma es su Documento en linea, pues tiene similitudes con el Word de office, pero en línea. En primer lugar, es un recurso que permite no cargar archivos sino crear un documento en la red, lo que evita que se multipliquen archivos de forma innecesaria e infinita. En segundo lugar, es una herramienta para compartir ideas en un entorno, sobre todo, público. Esto significa que permite exponer ideas para que sean evaluadas en forma grupal. En tercer lugar, en efecto es una herramienta ideal para trabajo 
colaborativo y cooperativo. Esto se debe a que cualquier integrante del grupo puede realizar la edición del contenido sin el riesgo de que se cree otro y se pierda lo creado. Por último, se trata de una herramienta para el aprendizaje grupal. No es un recurso aislado de los grupos de Facebook sino plenamente interactivo e integrativo (López, Flores y Espinoza, 2015).

Este tratamiento académico que diversos investigadores han hecho en torno a Facebook impacta mucho en los procesos de aprendizaje de la escritura académica, sobre todo para escritores novatos, quienes se familiarizan con esta plataforma como herramienta para adentrarse en la escritura universitaria. Un estudio donde se resaltan los rasgos de escritura en este entorno es el de Carvajal (2017), quien describió algunas características de este tipo de práctica diferenciándola de la escritura en papel. Por ejemplo, resaltó los rasgos translingüísticos y paralingüisticos dominantes, el uso frecuente de los emoticones y demás simbolos no verbales, la ligereza de las normas gramaticales y ortográficas y el empleo de otros recursos de inserción que, pese a todo, no afectan la coherencia del mensaje publicado.

La planificación textual, si bien es una etapa del proceso de escritura, es la que mayores dificultades genera en los estudiantes a la hora de pretender escribir (Gallego y Rodríguez, 2015). Elaborar planes que faciliten el adecuado desarrollo de composición de un texto académico demanda no solo tiempo sino también lectura y asesoramiento, pues implica "aterrizar" las ideas del escritor novato en constructos guía (Flower y Hayes, 1996). La planificación es, en tal sentido, aquella fase previa a la escritura y reescritura en la que se organiza la información sobre la cual se desea explicar (Moscol, 2018). Asimismo es necesaria para quien escribe porque integra estrategias guia para futuras experiencias de escritura. La planificación viene a ser, entonces, la representación de una actividad basada en un procedimiento de análisis e interpretación, en cuyo núcleo se transforma, mediante un filtro de contenido público, la situación retórica en un acto de escritura privado. Es decir, es el puente entre el nivel de las puras ideas y el texto concreto, por ende, es la primera forma de procesar las ideas en el largo proceso del aprendizaje de la escritura.

Por ello, según Arroyo y Salvador (2005) este tipo de planificación es considerado abstracto, y puede ser con o sin contenido dependiendo del grado de experticia del escritor. En el caso de escritores novatos, siempre se desarrollan esquemas de redacción detallados debido a la capacidad limitada de abstracción de los escritores novatos. En ese sentido, el esquema de redacción en este nivel posee una carga importante de información, expresada con ideas concretas y guias, por eso siempre es exhaustivo, coherente y jerárquico (Aguirre, 2017). Tomando en cuenta lo antedicho, la planificación textual para los escritores novatos debe poseer un contenido solvente de ideas, una organización jerárquica que demuestre capacidad de deducción y una formulación de estas expresadas con un lenguaje claro y coherente, no muy alejada de la de un texto en sí. 


\section{Metodología}

\section{Diseño de investigación}

Fue una investigación cuasiexperimental pues según Ato, López y Benavente (2013) se manipuló la variable dependiente, es decir, las calificaciones de una prueba de planificación textual. Se trabajó con dos grupos: uno recibió el programa pedagógico en Facebook y el otro el programa regular de las sesiones de la UTP, en el que no se incluyó plataforma digital ni dinámica alguna de cooperación en la planificación. Asimismo, tuvo un enfoque cuantitativo porque se procesaron los valores numéricos del grupo de control y del experimento a fin de contrastarlos. También fue explicativo debido a que se buscó determinar los efectos del programa pedagógico y responder, directamente, a la problemática planteada.

\section{Población y muestra del estudio}

Se consideró trabajar con dos grupos seleccionados por conveniencia de una población de 751 estudiantes, los cuales cursaron la asignatura Nivelación de Redacción en el año 2019. Como se ha consignado, el grupo de control estuvo constituido por 25 estudiantes que llevaron el programa educativo diseñado por la UTP, la cual consistía en organizar la información de fuentes escritas de forma individual y sin plataforma digital. En cambio, al grupo experimental -conformado por la misma cantidad de alumnos- se le aplicó un programa pedagógico sobre el uso de Facebook para procesar información. Los criterios de selección para los grupos fueron los siguientes:

- Edad: promedio de 18 años.

- Condición académica: que no fueran ni alumnos becados ni libres.

- Turno de estudios: estudiantes matriculados en el turno matutino.

- Frecuencia del curso: todos cursaban por primera vez la asignatura.

- Docencia: fueron estudiantes de un solo docente.

\section{Técnica e instrumento}

Por una parte, para la recolección de los datos se utilizó como técnica la observación del desempeño para planificar textos. Ello se logró diseñando un instrumento utilizado en el ámbito universitario, las rúbricas, pues según Gatica y Uribarren (2013), "son guías precisas que valoran los aprendizajes y productos realizados" (p. 61). Por ello, para la presente investigación se elaboró una rúbrica que evaluó las tres dimensiones de desempeño para la producción del esquema de redacción propio de la planificación. 


\begin{tabular}{|c|c|c|c|c|}
\hline Dimensión & Indicadores & I & II & III \\
\hline \multirow{3}{*}{$\begin{array}{l}\text { Contenido } \\
\text { (8 puntos) }\end{array}$} & $\begin{array}{c}\text { Tema } \\
\text { (2 puntos) }\end{array}$ & $\begin{array}{l}\text { El esquema está } \\
\text { basado en un tema } \\
\text { genérico expresado } \\
\text { en el título. Asimismo } \\
\text { solo se evidencia una } \\
\text { particularidad de la } \\
\text { delimitación, es decir, } \\
\text { solo usa el lugar, el } \\
\text { tiempo o el aspecto. } \\
\text { (0.5 puntos) }\end{array}$ & $\begin{array}{l}\text { El esquema está } \\
\text { basado en un tema casi } \\
\text { delimitado expresado, } \\
\text { además, en el título. } \\
\text { Asimismo solo considera } \\
\text { dos particularidades de } \\
\text { la delimitación. } \\
\text { (1 punto) }\end{array}$ & $\begin{array}{l}\text { El esquema está } \\
\text { basado en un tema } \\
\text { completamente } \\
\text { delimitado expresado } \\
\text { en el título. Asimismo } \\
\text { se evidencian todas las } \\
\text { particularidades de la } \\
\text { delimitación, es decir, } \\
\text { usa el lugar, el tiempo y } \\
\text { el aspecto. } \\
\text { (2 puntos) }\end{array}$ \\
\hline & $\begin{array}{l}\text { Pertinencia } \\
\text { (3 puntos) }\end{array}$ & $\begin{array}{l}\text { La información del } \\
\text { esquema es poco } \\
\text { pertinente con } \\
\text { respecto al tema de } \\
\text { investigación. } \\
\text { (0.5 puntos) }\end{array}$ & $\begin{array}{l}\text { La información del } \\
\text { esquema, en algunas } \\
\text { ocasiones, es pertinente } \\
\text { con respecto al tema de } \\
\text { investigación. } \\
\text { (1.5 puntos) }\end{array}$ & $\begin{array}{l}\text { La información } \\
\text { del esquema es } \\
\text { completamente } \\
\text { pertinente con respecto } \\
\text { al tema de investigación. } \\
\text { (3 puntos) }\end{array}$ \\
\hline & $\begin{array}{l}\text { Suficiencia } \\
\text { (3 puntos) }\end{array}$ & $\begin{array}{l}\text { La información } \\
\text { es genérica en } \\
\text { el esquema. Es } \\
\text { decir, las ideas en } \\
\text { todos los niveles } \\
\text { son escasamente } \\
\text { detalladas. } \\
\text { (0.5 puntos) }\end{array}$ & $\begin{array}{l}\text { La información es, en } \\
\text { algunas ocasiones, } \\
\text { suficiente en el } \\
\text { esquema. Es decir, } \\
\text { las ideas en todos los } \\
\text { niveles son, a veces, } \\
\text { detalladas. } \\
\text { (1.5 puntos) }\end{array}$ & $\begin{array}{l}\text { La información es } \\
\text { completamente } \\
\text { suficiente en el esquema. } \\
\text { Es decir, las ideas en } \\
\text { todos los niveles son } \\
\text { siempre detalladas. } \\
\text { (3 puntos) }\end{array}$ \\
\hline \multirow{3}{*}{$\begin{array}{l}\text { Organización } \\
\text { (8 puntos) }\end{array}$} & $\begin{array}{c}\text { Ideas } \\
\text { principales } \\
\text { (2 puntos) }\end{array}$ & $\begin{array}{l}\text { En el esquema, la } \\
\text { idea principal engloba } \\
\text { escasamente el tema } \\
\text { de investigación. } \\
\text { (0.5 puntos) }\end{array}$ & $\begin{array}{l}\text { En el esquema, la } \\
\text { idea principal engloba } \\
\text { parcialmente el tema de } \\
\text { investigación. } \\
\text { (1 punto) }\end{array}$ & $\begin{array}{l}\text { En el esquema, la } \\
\text { idea principal engloba } \\
\text { completamente el tema } \\
\text { de investigación. } \\
\text { (2 puntos) }\end{array}$ \\
\hline & $\begin{array}{c}\text { Ideas } \\
\text { secundarias } \\
\text { (3 puntos) }\end{array}$ & $\begin{array}{l}\text { En el esquema, las } \\
\text { ideas secundarias } \\
\text { evidencian mínima } \\
\text { relación lógica con la } \\
\text { idea principal. } \\
\text { (0.5 puntos) }\end{array}$ & $\begin{array}{l}\text { En el esquema, las ideas } \\
\text { secundarias evidencian } \\
\text { relativa relación lógica } \\
\text { con la idea principal. } \\
\text { Es decir, muestran } \\
\text { una clasificación } \\
\text { relativamente coherente } \\
\text { con la idea principal. } \\
\text { (1.5 puntos) }\end{array}$ & $\begin{array}{l}\text { En el esquema, las ideas } \\
\text { secundarias evidencian } \\
\text { completamente una } \\
\text { relación lógica con } \\
\text { la idea principal. Es } \\
\text { decir, muestran una } \\
\text { clasificación totalmente } \\
\text { coherente con la idea } \\
\text { principal. } \\
\text { (3 puntos) }\end{array}$ \\
\hline & $\begin{array}{l}\text { Ideas detalle } \\
\text { (3 puntos) }\end{array}$ & $\begin{array}{l}\text { En el esquema, } \\
\text { las ideas detalle } \\
\text { fundamentan poco } \\
\text { las ideas secundarias. } \\
\text { (0.5 puntos) }\end{array}$ & $\begin{array}{l}\text { En el esquema, las ideas } \\
\text { detalle fundamentan } \\
\text { relativamente las ideas } \\
\text { secundarias. } \\
\text { (1.5 puntos) }\end{array}$ & $\begin{array}{l}\text { En el esquema, las ideas } \\
\text { detalle fundamentan } \\
\text { completamente las ideas } \\
\text { secundarias. } \\
\text { (3 puntos) }\end{array}$ \\
\hline \multirow[t]{2}{*}{$\begin{array}{c}\text { Formulación (4 } \\
\text { puntos) }\end{array}$} & $\begin{array}{l}\text { Gramática } \\
\text { (2 puntos) }\end{array}$ & $\begin{array}{l}\text { Los enunciados } \\
\text { formulados en el } \\
\text { esquema son poco } \\
\text { coherentes. Es decir, } \\
\text { los enunciados casi } \\
\text { nunca se entienden. } \\
\text { (0.5 puntos) }\end{array}$ & $\begin{array}{l}\text { Los enunciados } \\
\text { formulados en el } \\
\text { esquema, en algunas } \\
\text { ocasiones, son } \\
\text { coherentes. Es decir, los } \\
\text { enunciados, a veces, se } \\
\text { entienden. } \\
\text { (1 punto) }\end{array}$ & $\begin{array}{l}\text { Los enunciados } \\
\text { formulados en } \\
\text { el esquema son } \\
\text { completamente } \\
\text { coherentes. Es decir, los } \\
\text { enunciados siempre se } \\
\text { entienden. } \\
\text { (2 puntos) }\end{array}$ \\
\hline & $\begin{array}{l}\text { Ortografía } \\
\text { (2 puntos) }\end{array}$ & $\begin{array}{l}\text { Los enunciados } \\
\text { formulados en el } \\
\text { esquema presentan } \\
\text { máximo } 8 \text { errores. } \\
\text { (0.5 puntos) }\end{array}$ & $\begin{array}{l}\text { Los enunciados } \\
\text { formulados en el } \\
\text { esquema presentan } \\
\text { máximo } 4 \text { errores. } \\
\text { (1 punto) }\end{array}$ & $\begin{array}{l}\text { Los enunciados } \\
\text { formulados en el } \\
\text { esquema presentan } \\
\text { máximo } 2 \text { errores. } \\
\text { (2 puntos) }\end{array}$ \\
\hline
\end{tabular}




\begin{tabular}{|c|c|c|}
\hline $\begin{array}{l}\text { Niveles de } \\
\text { aprendizaje }\end{array}$ & $\begin{array}{l}\text { Rango de } \\
\text { puntuación }\end{array}$ & Significado \\
\hline Destacado & $\begin{array}{l}\text { Más de } 17 \text { y hasta } \\
20\end{array}$ & $\begin{array}{l}\text { Cumple con planificar con mucha eficiencia el texto, por lo que el } \\
\text { esquema presenta ideas pertinentes, suficientes y de acuerdo con } \\
\text { el tema elegido, además ordenadas con jerarquía y gramatical y } \\
\text { ortográficamente bien escritas. }\end{array}$ \\
\hline Esperado & Más de 14 y hasta 17 & $\begin{array}{l}\text { Cumple con planificar con mínimos errores el texto, por lo que el } \\
\text { esquema presenta algunos errores en la pertinencia, la suficiencia } \\
\text { y el tema, además en el orden jerárquico y en la claridad de ideas. }\end{array}$ \\
\hline Estándar & Más de 11 y hasta 14 & $\begin{array}{l}\text { Cumple con planificar con varios errores el texto, por lo que el } \\
\text { esquema presenta errores en dos de los criterios. }\end{array}$ \\
\hline Básico & Hasta 11 & $\begin{array}{l}\text { No cumple con planificar eficientemente el texto, por lo que el } \\
\text { esquema presenta muchos errores en la pertinencia, la suficiencia } \\
\text { y el tema, además en el orden jerárquico y en la expresión } \\
\text { gramatical y ortográfica. }\end{array}$ \\
\hline
\end{tabular}

Por otra parte, para la validez de este instrumento se utilizó la validez por contenido por opinión de diez expertos en educación y TIC y los resultados, a través de la prueba binomial, arrojaron valores de 0.008. Asimismo se buscó la validez por constructo, por lo que se hizo el análisis factorial procedimental. De acuerdo con el análisis, la medida de Kaiser-Meyer-Olkin de adecuación de muestreo fue de 0.676, cercana al valor de aceptación, y el nivel de significancia de la prueba de esfericidad de Bartlett fue de 0,000, valores que evidenciaron la condición de aceptabilidad para el análisis factorial. De tal forma se observó que con 3 factores se obtuvo un porcentaje acumulado de varianza total de 55.63\%. Asimismo se buscó la fiabilidad del instrumento por equivalencia a través del coeficiente de correlación intraclase (CCI). Los resultados en el SPPS-25 concluyeron que el valor de correlación promedio de los valores asignados por dos observadores fue de 0.822. Considerando la clasificación asumida por Mandeville (2005) el valor se encuentra, entonces, entre 0.8 y 1 , lo que significa que el instrumento se prestó para evaluar con precisión el desempeño propuesto gracias a que existió equivalencia óptima entre los observadores.

\section{Programación del grupo experimental}

El grupo de control realizó actividades programadas por la UTP. Estas actividades estuvieron enfocadas en la esquematización de ideas a través de la lectura de fuentes escritas, en tiempos cronometrados y de forma individual, además de escritas en papel. Al final de la séptima semana se aplicó una prueba final de planificación. El grupo experimental, en cambio, realizó actividades cooperativas de planificación textual en 3 etapas, tarea que comprendió las 7 primeras semanas de clase de las 16 del periodo de estudio, tal como se detalla a continuación.

- Etapa de preparación

comprendió las dos primeras semanas de clase. En ese periodo los estudiantes se integraron al grupo de Facebook creado por el docente para la sección. Lo hicieron por medio de sus cuentas personales. Asimismo el docente explicó sobre el uso de la plataforma y sus recursos para el desarrollo del trabajo cooperativo. Con la orientación adecuada los estudiantes crearon su Documento en linea en Facebook, se agruparon en subgrupos de 5 miembros y anotaron sus temas generales afines de acuerdo con sus preferencias académicas. Luego cada grupo insertó en la portada del Documento en linea una imagen referencial al tema 
elegido. Como último proceso de esta etapa los estudiantes socializaron sus trabajos en el grupo de Facebook para que todos los de esa sección pudieran valorar la propuesta a través de los comentarios. Este tipo de valoración se enfocó en resaltar tanto las virtudes del tema y de la imagen como sus limitaciones a través de comentarios verbales y no verbales tales como los emoticones. Luego de ello, el grupo retroalimentado revisó los comentarios y los consideró para la mejora del trabajo de planificación. La edición se realizó en el mismo documento en línea creado por cada subgrupo.

\section{- Etapa de investigación}

Comprendió la tercera y la cuarta semana de clases. En ese periodo los estudiantes investigaron sobre su tema en Internet yjustificaron la elección de las fuentes seleccionadas en breves sumillas. Los documentos recopilados (videos, imágenes y textos) fueron insertados, a través de los hipervínculos, en el documento en línea ya creado por el subgrupo. Asimismo cada integrante las sumilló. Como último proceso de esta etapa los estudiantes socializaron sus documentos en línea al grupo de Facebook para que todos los miembros de la sección pudieran valorar la propuesta a través de los comentarios. Este tipo de valoración se enfocó en resaltar la pertinencia de las fuentes de información y la precisión de la sumilla así como sus limitaciones a través de comentarios verbales y no verbales a fin de que el autor, luego de su análisis, pudiera considerar la apreciación del grupo y, con ello, mejorar el trabajo de planificación.

- Etapa de producción

En las tres siguientes semanas de clase, con todos los productos previos, los estudiantes diseñaron el esquema de redacción a partir de la información recopilada y procesada considerando el contenido, la estructura y la formulación de ideas. Este trabajo fue realizado en el documento en línea ya creado desde hacía semanas. Lo cargaron como imagen, video o en enlace. Como último proceso de esta etapa los estudiantes socializaron sus documentos en línea en el grupo de Facebook para que todos los miembros de la sección pudieran valorar la propuesta a través de comentarios. Este tipo de valoración se enfocó en evaluar si el contenido fue pertinente y suficiente, si la organización cumplió con un sistema jerárquico y si las ideas se expresaron con claridad gramatical y ortográfica a través de comentarios verbales y no verbales. Luego de ello el grupo retroalimentado revisó los comentarios y los consideró para la mejora del trabajo de planificación. Finalmente, cada grupo presentó su trabajo de planificación al docente para su evaluación final. 


\section{Resultados}

Al grupo de control y al experimental se les aplicó una prueba de entrada que consistió en elaborar un esquema de ideas a partir de un tema de elección personal, de algunas fuentes propuestas y propias, y de diseño propio. Se realizó en la primera semana de clases. Otra prueba similar se aplicó al finalizar la séptima semana.

\section{Resultados del grupo de control}

Tabla 3. Medias de los datos del pretest y postest del grupo de control

\begin{tabular}{|c|c|c|c|}
\hline & Unidades & Media del pretest & Media del postest \\
\hline $\begin{array}{c}\text { Variable: } \\
\text { Planificación textual }\end{array}$ & 25 & 6.16 & 12.23 \\
\hline Dimensión 1: Contenido & 25 & 1.73 & 4.31 \\
\hline Dimensión 2: Estructura & 25 & 1.71 & 4.96 \\
\hline Dimensión 3: Formulación & 25 & 2.72 & 3.46 \\
\hline
\end{tabular}

Los resultados del pretest indicaron que las notas obtenidas en la variable estudiada oscilan entre 3.5 y 9.75, donde 4.5. 5.75 y 7 son las de mayor frecuencia. Eso difirió de las calificaciones del postest que fueron de 9.25 a 15. En esta última prueba, la mayor cantidad de puntuación (2 datos) correspondió a 11.5. Por esas razones ambas muestras presentaron medias que ubicaron el desempeño de los estudiantes en niveles de aprendizaje distintos. En el pretest, la media fue de 6.16 y en postest de 12.23, por lo que corresponderían al nivel Básico y Estándar, respectivamente. Por un lado, esto significa que los estudiantes ingresaron a la universidad con serios problemas para planificar información antes de escribir textos académicos. Vale decir que muchos de ellos no supieron seleccionar ideas pertinentes y suficientes de acuerdo con sus temas de trabajo. Tampoco supieron ordenar sus ideas con jerarquía y menos aun plantearlas con claridad de acuerdo con las normas gramaticales y ortográficas. Por otro lado, pese a que se aplicó el programa regular de la UTP, los estudiantes pudieron aprender a elegir, ordenar y formular ideas pero no con mucha solvencia. Se observaron errores en dos criterios, concretamente en el contenido (ideas poco pertinentes y suficientes) y en el orden de las ideas (sin ideas principales), de ahí que se haya obtenido la mitad del puntaje esperado.

Respecto a la dimensión Contenido se halló que, en el pretest, las calificaciones oscilaron entre 0.75 y 3 respecto a los 8 puntos que correspondian a esta dimensión. Asimismo, la frecuencia mayor fue de 1.5 (5 datos) y, en el postest, de entre 3 y 5.75, cuya mayor frecuencia fue 4, lo que dio a entender que existió una mayor tendencia de aciertos en el postest, aunque fue la mitad del valor. Por ello se observó una diferencia descriptiva entre sus medias, una de 2.58 puntos a favor de esta última. En la dimensión Estructura los resultados indicaron que los puntos en el pretest fluctuaron entre 0.75 a 3.25 puntos, y su frecuencia mayor recayó en 1.25 puntos, en comparación con la recurrencia de datos en el postest, que fueron de 4.5 y 5.25. Si bien no se obtuvieron valores cercanos a lo esperado (8 puntos), sí hubo una diferencia importante en ambas pruebas: 1.71 y 4.96 fueron sus medias, respectivamente. Acerca de la dimensión Formulación, 3 fue la calificación más 
frecuente en el pretest y, en el postest, de 2, 3 y 3.5 puntos, de ahi que la diferencia de sus medias (0.24) haya sido cercana al menor. Por lo mismo, es la que menos dificultades ha generado para los estudiantes en sus tareas de planificación textual si se compara con las anteriores dimensiones.

\section{Resultados del grupo experimental}

Tabla 4. Medias de los datos del pretest y del postest del grupo experimental

\begin{tabular}{|c|c|c|c|}
\hline & Unidades & $\begin{array}{c}\text { Media del } \\
\text { pretest }\end{array}$ & Media del postest \\
\hline $\begin{array}{c}\text { Variable: } \\
\text { Planificación textual }\end{array}$ & 25 & 6.04 & 17.53 \\
\hline Dimensión 1: Contenido & 25 & 1.79 & 7.04 \\
\hline Dimensión 2: Estructura & 25 & 1.67 & 7.02 \\
\hline Dimensión 3: Formulación & 25 & 2.58 & 3.47 \\
\hline
\end{tabular}

Los resultados del pretest indicaron que las calificaciones obtenidas en la variable Planificación textual oscilaron entre 3 y 9.5 puntos, donde 5.5, 6 y 6.5 fueron los de mayor frecuencia. Eso difirió de las calificaciones del postest que fueron desde el 8.75 hasta 20. En esta última prueba, la mayor cantidad de puntuación (3 datos) correspondió a 18.75. Por ello ambas pruebas presentaron medias que ubicaron el desempeño de los estudiantes en niveles de aprendizaje diferentes. En el pretest, la media fue de 6.04 y, en el postest, de 17.53, por lo que corresponderian al nivel Básico y Destacado, respectivamente. Esto significa que, por un lado, la media de los puntajes correspondía al mismo nivel pero los puntajes individuales de los 25 sujetos fueron diferentes en cuanto a su distribución. Por otro lado, los estudiantes pudieron aprender a elegir con pertinencia sus temas de trabajo y escribirlos con suficiencia y pertinencia, además de ordenar con jerarquía las ideas y formular con una solvencia gramatical y ortográfica las ideas.

Respecto a la dimensión Contenido se indicó que, en el pretest, las calificaciones oscilaron entre 0.75 y 3.25, respecto a los 8 puntos que le correspondian. Asimismo, la frecuencia mayor fue de 1 y 2 (4 datos para cada valor) y, en el postest, de entre 6 y 8 , cuya mayor frecuencia (6 datos) fue 7.5, lo que dio a entender que existió una mayor tendencia de aciertos en el postest. Por ello se observó una diferencia descriptiva entre sus medias, una de 5.25 puntos a favor de la última prueba. En la dimensión Estructura los resultados indicaron que las notas oscilaron entre 0.75 a 2.75 puntos, y su frecuencia mayor recayó en 1.75 puntos en comparación con la recurrencia de datos en el postest que fue de 7.25 y 7.5 . Si bien no se obtuvieron valores cercanos a lo estimado (8 puntos), sí hubo una diferencia importante en ambas pruebas: 1.67 y 7.02 fueron sus medias, respectivamente. Acerca de la dimensión Formulación, la calificación más frecuente en el pretest fue de 2.5 y, en el postest, de 3.5 y 4 (4 datos para cada valor) de los 4 puntos esperados, de ahí que la diferencia de sus medias (0.89) haya sido mínima. Por dicha razón fue la que menos dificultades ha generado a los estudiantes en sus tareas de planificación textual si se compara con las anteriores dimensiones. 


\section{Prueba de normalidad}

Las unidades muestrales de esta investigación fueron menores a 50. Por ello, a través de la prueba Shapiro-Wilk se concluyó que los datos analizados tuvieron una distribución normal. Así, en el grupo de control, para la variable Planificación textual el valor de $p$ fue 0.593 y 0.916 en el pretest y postest, respectivamente. En las dimensiones Contenido, Estructura y Formulación del mismo grupo dichos valores fueron los siguientes: $0.120,0.275$ y 0.288 en el pretest y, 0.472, 0.641 y 0.151 en el postest. En el grupo experimental, los valores de normalidad fueron, en el pretest, 0.209 para la variable y $0.231,0.700$ y 0.723 para sus dimensiones, y en el postest, 0.209 para la variable y $0.100,0.102$ y 0.090 para sus dimensiones.

\section{Prueba de hipótesis}

Considerando la normalidad de los datos de la variable y de las dimensiones, se utilizó la Prueba T-Student para el contraste de hipótesis. Se consideraron las siguientes condiciones:

- Si p valor es menor a 0.05 se rechaza la hipótesis nula $\left(\mathrm{H}_{0}\right)$, es decir, se rechaza la igualdad entre los valores de las dos variables comparadas y se acepta la hipótesis alternativa $\left(H_{1}\right)$, es decir, que existe diferencia entre ambas.

- Si p valor es mayor a 0.05 no se rechaza la hipótesis nula $\left(H_{0}\right)$, es decir, se asume que los valores de las variables expresan igualdad.

Tabla 5. Prueba T de Student para muestras relacionadas

\begin{tabular}{|c|c|c|c|c|c|c|c|c|}
\hline \multirow{2}{*}{$\begin{array}{c}\text { Grupos } \\
\text { comparados }\end{array}$} & \multirow{2}{*}{$\begin{array}{c}\text { Dimensiones/ } \\
\text { Variables }\end{array}$} & & \multicolumn{4}{|c|}{ Diferencias emparejadas } & \multirow[b]{2}{*}{ t } & \multirow[b]{2}{*}{ Sig. } \\
\hline & & Media & Desviación & $\begin{array}{c}\text { Error } \\
\text { promedio }\end{array}$ & \multicolumn{2}{|c|}{$\begin{array}{l}95 \text { \% de intervalo } \\
\text { de confianza de } \\
\text { la diferencia }\end{array}$} & & \\
\hline \multirow{4}{*}{$\begin{array}{l}\text { Pretest vs } \\
\text { Postest }\end{array}$} & $\begin{array}{l}\text { Variable: } \\
\text { Planificación } \\
\text { textual }\end{array}$ & 11.49 & .48670 &, 09734 & 11,2891 & 11,6909 & 118,04 &, 000 \\
\hline & $\begin{array}{l}\text { Dimensión } \quad 1 \text { : } \\
\text { Contenido }\end{array}$ & 5.25 & ,22822 & .04564 & 5,1558 & 5,3442 & 115,02 &, 000 \\
\hline & $\begin{array}{l}\text { Dimensión 2: } \\
\text { Estructura }\end{array}$ & 5.35 & ,27951 & ,05590 & 5,2346 & 5,4653 & 95,70 &, 000 \\
\hline & $\begin{array}{l}\text { Dimensión } 3: \\
\text { Formulación }\end{array}$ & 0.89 & .25083 & .05017 &, 7864 & 9935 & 17,74 &, 000 \\
\hline
\end{tabular}

Nota: Resultados obtenidos del SPSS-25.

Según la tabla 5, la diferencia descriptiva entre el pretest y postest entre las medias del grupo experimental de la variable Planificación textual fue de 11.49 a un nivel de significancia menor a 0.05. Por ello se rechazó la hipótesis nula $\left(H_{0}\right)$ y se asumió que la hipótesis alterna $\left(H_{1}\right)$ fue válida. Con esto se pudo asumir, entonces, que el valor de $p(0.000)$ fue evidencia para afirmar que si existen diferencias significativas entre ambos momentos del grupo experimental. 
De la misma manera, la dimensión Contenido del grupo experimental fue de 5.25 a un nivel de significancia menor a 0.05. Por ese motivo, la hipótesis nula $\left(\mathrm{H}_{0}\right)$ fue rechazada y se asumió como válida la hipótesis alterna $\left(H_{1}\right)$, la cual afirma la diferencia entre ambos valores. Respecto a la dimensión Estructura de la misma variable el resultado de contraste fue de 5.35 a un nivel de significancia menor a 0.05 . Por eso, la hipótesis nula $\left(H_{0}\right)$ fue rechazada y la hipótesis alterna $\left(H_{1}\right)$ fue considerada válida, lo cual se interpretó a través de la siguiente afirmación: el valor de $p(0.000)$ fue un hecho probabilístico para estimar que sí existe diferencia significativa. Respecto a la dimensión Formulación, el resultado de contraste entre ambas muestras fue de 0.89 a un nivel de significancia menor a 0.05. Por ese valor se rechazó la hipótesis nula $\left(\mathrm{H}_{0}\right)$ y se asumió que la hipótesis alterna $\left(\mathrm{H}_{1}\right)$ fue válida.

Tabla 6. Prueba T de Student para muestras independientes

\begin{tabular}{|c|c|c|c|c|c|c|c|c|}
\hline \multirow{2}{*}{$\begin{array}{c}\text { Grupos } \\
\text { comparados }\end{array}$} & \multirow{2}{*}{$\begin{array}{c}\text { Dimensiones/ } \\
\text { Variables }\end{array}$} & $\begin{array}{l}\text { Prueba de } \\
\text { Levene de } \\
\text { igualdad }\end{array}$ & \multicolumn{6}{|c|}{ Prueba T para la igualdad de medias } \\
\hline & & Sig. & T & Sig. & $\begin{array}{l}\text { Diferencias } \\
\text { de medias }\end{array}$ & $\begin{array}{l}\text { Diferencia } \\
\text { de error } \\
\text { estándar }\end{array}$ & $\begin{array}{r}95 \% \text { d } \\
\text { de con } \\
\text { diff }\end{array}$ & $\begin{array}{l}\text { itervalo } \\
\text { nza de la } \\
\text { ncia }\end{array}$ \\
\hline \multirow{4}{*}{$\begin{array}{l}\text { Experimento } \\
\text { vs Control }\end{array}$} & $\begin{array}{c}\text { Variable: } \\
\text { Planificación } \\
\text { textual }\end{array}$ &, 711 & 10,59 & .000 & 5.30 & .50047 & 4,2937 & 6,3062 \\
\hline & $\begin{array}{l}\text { Dimensión 1: } \\
\text { Contenido }\end{array}$ &, 178 & 13,44 & .000 & 2,73 &, 20308 & 2,3216 & 3,1383 \\
\hline & $\begin{array}{c}\text { Dimensión 2: } \\
\text { Estructura }\end{array}$ & 617 & 10,95 & .000 & 2,06 & 18797 & 1,6820 & 2,4379 \\
\hline & $\begin{array}{l}\text { Dimensión 3: } \\
\text { Formulación }\end{array}$ & .502 & 3,22 &, 002 & 0,51 &, 15811 & 1920 & 8279 \\
\hline
\end{tabular}

Nota: Resultados obtenidos del SPSS-25.

Según la tabla 6, la diferencia descriptiva entre el postest de la variable Planificación de textos académicos del grupo de control y experimental fue de 5.30 a un nivel de significancia menor a 0.05 . Por ello se rechazó la hipótesis nula $\left(\mathrm{H}_{0}\right)$ y se asumió que la hipótesis alterna $\left(H_{1}\right)$ fue válida. En ese sentido, se pudo asumir, entonces, que el valor de $p(0.000)$ fue evidencia para afirmar que sí existen diferencias entre el grupo de control y el experimental.

La misma tendencia probabilística resultó de los datos procesados de las dimensiones contrastadas. Primero, respecto a la dimensión Contenido, fue de 2.73 a un nivel de significancia menor a 0.05. Por ese motivo se rechazó la hipótesis nula $\left(\mathrm{H}_{0}\right)$ y se asumió que la hipótesis alterna $\left(\mathrm{H}_{1}\right)$ fue válida. Se pudo asumir, entonces, que el valor de $p(0.000)$ fue evidencia para afirmar diferencias significativas entre ambos grupos. Segundo, respecto a la dimensión Estructura, el resultado fue de 2.06 a un nivel de significancia menor a 0.05. Por esa razón se rechazó la hipótesis nula $\left(H_{0}\right)$ y se asumió la hipótesis alterna $\left(H_{1}\right)$ como válida. Tercero, respecto a la dimensión Formulación el valor obtenido fue 0.51 a un nivel de significancia menor a 0.05. Por ello se rechazó la hipótesis nula $\left(H_{0}\right)$ y se asumió a la hipótesis alterna $\left(\mathrm{H}_{1}\right)$ como la mejor. Se pudo asumir en ambos casos, entonces, que el valor de $p$ fue evidencia para afirmar que sí existe una diferencia considerable a favor del grupo experimental. 


\section{Conclusiones y discusión}

Las conclusiones de esta investigación van por la misma línea de ideas que los resultados aportados por González (2012), quien afirmó que Facebook no solo es una herramienta de comunicación muy versátil sino, además, un entorno para trabajar cooperativamente proyectos de investigación, y por Aristizábal, Becerra y Díaz (2018) y Carvajal (2017), quienes indicaron que este recurso virtual es útil para realizar actividades de escritura compartidas e hipertextualizadas.

Por ello, el presente trabajo tuvo como objetivo principal analizar los resultados del uso de Facebook obtenidos en una prueba de desempeño para planificar textos académicos en esquemas de redacción. De esa manera se buscó, en específico, identificar cómo esa plataforma afecta la calidad del contenido de la información, de la organización de las ideas y de la formulación de estas en los esquemas de redacción en el contexto del curso Nivelación de Redacción de la Universidad Tecnológica del Perú.

Respecto al objetivo principal, se concluye que gracias a que utilizó la red social en actividades de apoyo cooperativo, el estudiantado mejoró su desempeño para elaborar esquemas de redacción. Con este uso pedagógico llegó a alcanzar calificaciones de 17.53 en promedio, lo que equivalió, en el nivel de aprendizaje, a un Destacado, en comparación con el nivel de aprendizaje Estándar del grupo de control al cual no se aplicó experimento de uso de plataforma digital alguno. Esto significó que, según los valores descritos, el uso de esta plataforma resultó muy efectivo, no solo porque permitió que el estudiantado aprendiera a planificar ideas en esquemas de redacción considerando el contenido, el orden y la formulación de ideas sino, porque permitió, asimismo, que pudiera integrarse y desarrollar conocimiento en beneficio del grupo de manera más efectiva que el grupo de control. En este experimento la idea del trabajo individual en un curso de Humanidades fue cuestionada, al considerar el apoyo como modo de mejorar a los demás en doble forma, entre integrantes de subgrupos y grupos de sección. Aunque la virtud de esta herramienta ya la habia intuido González (2012) en su propuesta en entornos escolares, se considera que para potenciar la propuesta de esta investigación en la universidad en cuestión es necesario que paulatinamente se apliquen diseños de clase basados en herramientas digitales abiertas. Y esto debe realizarse, sobre todo, en cursos generales para, además de mejorar la actitud respecto a la competencia de la comunicación escrita, dar mayores facilidades al estudiantado que por diversos motivos dependen mucho del docente, se autoaislan del entorno y asumen la idea de competencia como disputa y no como oportunidad para desarrollarse.

Respecto al primer objetivo especifico se concluyó que en virtud de la cooperación en esta plataforma web el estudiantado pudo mejorar el nivel del contenido de su desempeño para elaborar esquemas de redacción. Con el uso pedagógico llegó a alcanzar, en promedio, valores de 7, en comparación con el grupo de control cuyo nivel de aprendizaje apenas fue por debajo de la mitad del valor, con apenas 4.31 en promedio. Esto indicó que el programa pudo ayudar, por un lado, a la recolección acumulada de información de los estudiantes en el entorno web y al procesamiento de la misma de forma más pertinente y suficiente que sin Facebook y la técnica de trabajo, y, por otro lado, ayudó a que el estudiantado pudiera socializar los productos presentados y asumir compromisos para mejorar. Este modo de trabajar ha permitido que los estudiantes se vieran beneficiados no solo en sus calificaciones sino también en considerar como necesario el apoyo del otro para proponer temas académicos y colocar ideas pertinentes y suficientes con el objetivo puntual. Sin embargo, pese al apoyo cooperativo, el estudiantado tuvo ciertos errores al colocar ideas pertinentes lo que generó que no se llegara al logro pleno. 
Respecto al segundo objetivo específico se concluyó que el desempeño de los estudiantes a la hora de ordenar ideas en esquemas de redacción mejoró, y esto sucedió debido a la intervención cooperativa en las actividades para ordenar las ideas principales, secundarias y detalle. Con el uso pedagógico, el estudiantado llegó a alcanzar valores de 7.02, el máximo de la dimensión, en comparación con el 4.96 del grupo de control. En tal sentido, las ideas principales y secundarias, que en el grupo de control no pudieron desarrollarse con solvencia, en el experimento pudieron colocarse con pertinencia gracias a esa doble valoración del subgrupo y del grupo de la sección, aunque se registró alguna limitación en la identificación y en el mejoramiento de las ideas principales. Esto indicó que en la producción de esquemas no es suficiente el apoyo del grupo. La mirada evaluativa del docente debe regularse de forma tal que pueda participar en momentos precisos en el proceso de esquematización.

Respecto al tercer objetivo específico se concluyó que la herramienta social, en efecto, no impactó significativamente en el desempeño para formular adecuadamente ideas en los esquemas de redacción. Si bien no fue significativo, se evidencian patrones que deben considerarse en el trabajo de escritura. La ortografía y la gramática se desarrollaron bajo presupuestos que dependieron de la experiencia educativa previa y no tanto del trabajo de planificación de ideas en plataformas virtuales o presenciales. Por ello, los valores tanto en el control como en el experimento fueron casi los mismos.

Nota: Aprobación final del artículo: editora responsable Mag. Verónica Zorrilla de San Martín.

Contribución de autoría: La elaboración del artículo es obra única del autor 


\section{Referencias bibliográficas}

AGUIRRE, M. (2017). Cómo leer y escribir en la universidad. Universidad Peruana de Ciencias Aplicadas.

ALARCÓN, E., SEPÚLVEDA, P. Y MADRID, D. (2018). Qué es y qué no es aprendizaje cooperativo. Revista de la Facultad de Educación de Albacete [Revista electrónica], 33 (1). https://dialnet.unirioja.es/servlet/articulo?codigo=6536516

ÁLVAREZ, M. (2017). La influencia de la red social Facebook en el lenguaje escrito de los estudiantes del grado octavo del colegio Jaime Garzón de Cúcuta (Tesis de licenciatura). Universidad Santo Tomas. Colombia.

ARISTIZÁBAL, Y., BECERRA, N. Y DÍAZ, D. (2018). La escritura como medio de comunicación en Facebook y su influencia en la vida de tres jóvenes (Tesis de licenciatura). Universidad de la Salle, Colombia.

ARROYO, R. Y SALVADOR, F. (2005). El proceso de planificación en la composición escrita de alumnos de educación primaria. Revista en Educación [Revista electrónical, 336. http://www.educacionyfp.gob.es/dam/jcr:b7c8c999-dffo4943-a4c4-7f162a1f431f/re33618-pdf.pdf

ATO, M., LÓPEZ, J. Y BENAVENTE, A. (2013). Un sistema de clasificación de los diseños de investigación en psicología. Anales de psicología [Revista electrónica], 29 (3). https://www.redalyc.org/pdf/167/16728244043.pdf

AZORÍN, C. (2018). El método de aprendizaje cooperativo y su aplicación en las aulas. Perfiles Educativos [Revista electrónica], 40 (161). http://www.iisue.unam. $\mathrm{mx} /$ perfiles/articulo/2018-161-el-metodo-de-aprendizaje-cooperativo-y-suaplicacion-en-las-aulas.pdf

CARVAJAL, G. (2017). Lectura y escritura en la red: ¿acceso a la información o dominio de la cultura escrita? Nexus Comunicación, 22, 138-157. DOl: https://doi. org/10.25100/nc.voi22.6244

DEL BARRIO, A. Y RUIZ, I. (2014). Los adolescentes y el uso de las redes sociales. Revista de Psicología [Revista electrónica], 1 (3). https://www.redalyc. org/pdf/3498/349851785056.pdf [Fecha de consulta: julio 10 de 2020].

FERNÁNDEZ DEL HARO, E. (2013). El trabajo en equipo mediante aprendizaje cooperativo. Curso de tutoría y orientación en la educación superior. Universidad de Granada. http://conexiones.dgire.unam.mx/wp-content/uploads/2017/og/ El-trabajo-en-equipo-mediante-aprendizaje-cooperativo-en-grupos.pdf

FLOWER, L. Y HAYES, J. (1996). Teoría de la redacción como proceso cognitivo. Textos en contexto. Asociación Internacional de Lectura.

FONSECA, O. (2015). Redes sociales y juventudes: uso de Facebook por jóvenes de México, Argentina y Colombia. (Tesis doctoral, España). https://riuma.uma. es/xmlui/bitstream/handle/10630/11548/TD_FONSECA_MANTILLA_Oscar. pdf? sequence $=1 \&$ isAllowed $=y$

GALlEGo, J. Y RODRíGuEZ, A. (2016). Caracteristica de la planificación de la escritura en estudiantes con Síndrome de Down. Revista Electrónica Actualidades Investigativas en Educación, 16(1), 1-29. https://revistas.ucr.ac.cr/index.php/aie/ article/view/22666 
GARCÍA, E., GARCÍA, A. Y REYES, J. (2014). Relación maestro alumno y sus implicaciones en el aprendizaje. Ra Ximhai, [Revista electrónica], 10 (5). https:// www.redalyc.org/articulo.oa?id $=46132134019$

GARZÓN, A. (2016). Influencia de las TIC en la escritura juvenil contemporánea. Actualidades pedagógicas, 68, 135-157. DOI: 10.19052/ap.3745

GATICA, F. Y URIBARREN, T. (2013). ¿Cómo elaborar una rúbrica? Investigación en educación médica [Revista electrónica], 2 (5). http://www.scielo.org.mx/scielo. php?script=sci_arttext\&pid=S2007-50572013000100010\&lng=es

GONZÁLEZ, P. (2012). Facebook, plataforma para crear actividades cooperativas. Actas de las IV Jornadas de Formación para Profesores de Español en Chipre. (1622). España. Universidad de Chipre (Editores). https://dialnet.unirioja.es/servlet/ libro?codigo $=572714$

GUDIÑO, S., LOZANO, F. Y FERNÁNDEZ, J. (2014). Uso de Facebook para la socialización del aprendizaje de una segunda lengua en nivel medio superior. Sinéctica [Revista electrónica], 42. http://www.scielo.org.mx/scielo. php?script=sci_arttext\&pid=S1665-109X2014000100008\&lng=es\&tlng=es

JOHNSON, D. W. Y JOHNSON, R. T. (1999). El aprendizaje cooperativo en el aula. España: Paidós.

KAGAN, S. (1985) Dimensions of cooperative classroom structures IDimensiones de las estructuras cooperativas del aulal. Springer. https://doi.org/10.1007/978-14899-3650-9_3

LÓPEZ, D., FLORES, K. Y ESPINOZA, A. (2015). Diversidad de usos de Facebook en la educación superior. Análisis desde un caso de estudio. Innoeduca, 1 (2), 106114. DOI: https://doi.org/10.20548/innoeduca.2015.v1i2.1040

MANDEVILLE, P. (2005). El coeficiente de correlación intraclase. Ciencia UANL [Revista electrónica], 8 (3). https://www.redalyc.org/pdf/402/40280322.pdf

MENDOZA, M. (2019). La comunicación escrita en Facebook y la redacción en la producción de textos explicativos que realizan los estudiantes de primer ciclo en el curso de Nivelación de Redacción en una universidad privada de Arequipa en el 2018 (Tesis de maestría). Universidad Tecnológica del Perú. http://repositorio. utp.edu.pe/bitstream/UTP/2502/1/Mary\%20Mendoza_Trabajo\%20de\%20 Investigacion_Maestria_2019.pdf

MOSCOL, D. (2018). Propuesta metodológica basada en el análisis textual para la enseñanza de la escritura académica. Comunicare: Revista de investigación de la Facultad de Humanidades, 4 (2), 23-31. DOI: https://doi.org/10.35383/educare. v2i7.77

NÚÑEZ, S., ZAMBRANO, A., PALACIO, J. Y MALDONADO, F. (2020). Juegos de negociación: estrategia para formación de competencias ciudadanas en universitarios. Educación y Educadores [Revista electrónica], 23 (2). https://educacionyeducadores.unisabana.edu.co/index.php/eye/article/ view/12995/5901 
PARRA, C., GONZÁLEZ-SICILIA, M. Y BELTRÁN, M. (2013). La creación de grupos en Facebook como apoyo a la enseñanza universitaria. Una aplicación en la materia de marketing. Congreso Internacional EDUTEC. Memoria: Educación y tecnologia: una oportunidad para impulsar. (1-13). Costa Rica. Escuela de Ciencias Exactas y Naturales. https://www.uned.ac.cr/academica/edutec/memoria/ ponencias/parra_sicilia_\%2obeltrang4.pdf

RAMÍREZ, S. (2013). La libertad de expresión del trabajador en Facebook y el poder disciplinario del empleador. Revista IUS, 7(31), 48-66. http://Www.scielo.org.mx/ scielo.php?script=sci_arttext\&pid=\$1870-21472013000100004\&lng=es\&tlng=es

RUEDA, R. Y GIRALDO, D. (2016). La imagen de perfil en Facebook: identidad y representación en esta red social. Revista Folios [Revista electrónica], 43. https:// www.redalyc.org/pdf/3459/345943442009.pdf

SIMANCA, F., BLANCO, F. Y CIFUENTES, C. (2019), Desarrollo de competencias lecto-escriturales mediante un prototipo de sistema de información. Revista Vinculos: Ciencia, Tecnología y Sociedad, 16 (1), 29-41. DOl: https://doi. org/10.14483/2322939X.15073

TORRES, M. Y YEPES, D. (2018). Aprendizaje cooperativo y TIC y su impacto en la adquisición del idioma inglés. Revista Mexicana de Investigación Educativa [Revista electrónica], 23 (78). http://www.scielo.org.mx/pdf/rmie/v23n78/14056666-rmie-23-78-861.pdf

VIVERO, N., TOALA, M. Y MACÍAS M. (2018). Elaprendizaje cooperativo en el proceso de enseñanza aprendizaje de la escritura del idioma académico inglés como lengua extranjera. Polo del Conocimiento [Revista electrónica], 3 (12). https:// www.mendeley.com/catalogue/e647a7ab-1ba3-3d80-9d3a-ecae5d3b9076/ 